“(C) 2018 IEEE. Personal use of this material is permitted. Permission from IEEE must be obtained for all other uses, in any current or future media, including reprinting/republishing this material for advertising or promotional purposes, creating new collective works, for resale or redistribution to servers or lists, or reuse of any copyrighted component of this work in other works." 


\title{
Construction and Performance of Quantum Burst Error Correction Codes for Correlated Errors
}

\author{
Jihao Fan*‡, Min-Hsiu Hsieh ${ }^{\dagger}$, Hanwu Chen ${ }^{\ddagger}, \mathrm{He} \mathrm{Chen}^{\S}$, and Yonghui $\mathrm{Li}^{\S}$ \\ *Nanjing Institute of Technology, Nanjing, Jiangsu, China \\ †University of Technology Sydney, Australia \\ ${ }^{\ddagger}$ Southeast University, Nanjing, Jiangsu, China \\ $\S$ University of Sydney, Australia \\ jihao.fan@outlook.com, min-hsiu.hsieh@uts.edu.au,hw_chen@seu.edu.cn, \{he.chen, yonghui.li\}@sydney.edu.au
}

\begin{abstract}
In practical communication and computation systems, errors occur predominantly in adjacent positions rather than in a random manner. In this paper, we develop a stabilizer formalism for quantum burst error correction codes (QBECC) to combat such error patterns in the quantum regime. Our contributions are as follows. Firstly, we derive an upper bound for the correctable burst errors of QBECCs, the quantum Reiger bound (QRB). This bound generalizes the quantum Singleton bound for standard quantum error correction codes (QECCs). Secondly, we propose two constructions of QBECCs: one by heuristic computer search and the other by concatenating two quantum tensor product codes (QTPCs). We obtain several new QBECCs with better parameters than existing codes with the same coding length. Moreover, some of the constructed codes can saturate the quantum Reiger bounds. Finally, we perform numerical experiments for our constructed codes over Markovian correlated depolarizing quantum memory channels, and show that QBECCs indeed outperform standard QECCs in this scenario.
\end{abstract}

\section{INTRODUCTION}

In classical information theory, there are mainly two types of error models: the independent noise model proposed by Shannon and the adversarial noise model considered by Hamming. Errors in these two models are usually called random errors and burst errors. Correspondingly, there are random errorcorrecting codes (RECC) and burst error-correcting codes (BECC) to deal with these two different types of errors [1]. In reality, channels tend to introduce errors which are localized in a short interval, i.e., the burst errors. These errors could be commonly found in communication systems and storage mediums, as a result of a stroke of lightning in wireless channels or scratch on a storage disc.

In the quantum regime, quantum errors can be independent or correlated in space and time. Hence there are counterparts of quantum random error-correcting codes (see, e.g., [2]-[6]) and quantum burst error-correcting codes [7]-[10]. Analogous to the classical case, quantum channels commonly have memory [11] or introduce errors which are localized [12], i.e., quantum burst errors. Vatan et al. [8] first considered spatially correlated qubit errors and constructed families of QBECCs using CSS construction [3], [4]. However, CSS construction yields QBECCs with inferior code rate. In [9], a quantum interleaver for QBECCs was proposed so that long QBECCs could be produced from short ones. However, this method highly relies on short efficient QBECCs, which are lacking at this moment. In [10], QBECCs of length up to 51 were found using computer search.

The construction and investigation of QBECCs have received far less attention, compared to the development of standard QECCs or entanglement-assisted QECCs [5], [6], [13]-[17]. Many important questions remain open. Currently, there is no general upper bound for correctable quantum burst errors, analygous to the classical Reiger bound: $n-k \geq 2 \ell$, of an $[n, k]$ classical BECC, where $n$ is the code length, $k$ is the message size, and $\ell$ is the correctable length of burst errors. In addition, there is an interesting class of quantum codes, called degenerate codes, that have no classical correspondences. They can potentially store more quantum information or correct more quantum errors than nondegenerate codes. However, degenerate QBECCs have never been explored.

In this paper we generalize the theory of standard QECCs to QBECCs. We develop the stabilizer formalism for QBECCs and prove the corresponding quantum Reiger bound: $n-k \geq$ $4 \ell$, for an $[[n, k]]$ QBECC that corrects a quantum burst error of length $\ell$ or less. The quantum Reiger bound further generalizes the quantum Singleton bound in QECCs. We obtain many new QBECCs in the stabilizer formalism via computer heuristic search, and these codes are better than existing QBECCs with the same code lengths. We show that the burst error-correcting abilities of most of these codes can achieve the quantum Reiger bound and thus are perfect codes. In particular, several of our constructed QBECCs, that attain the quantum Reiger bound, are degenerate codes. Additionally, we propose a new concatenation construction of long QBECCs from two short component codes based on the quantum tensor product code structure [7] and the interleaving technique. Since only one of the component codes of QTPCs needs to satisfy the dual containing constraint, this construction method can largely facilitate the systematical construction of QBECCs. Finally, we perform numerical experiments on two of our constructed QBECCs by measuring the entanglement fidelity over Markovian correlated depolarizing quantum memory channels [12]. It is known that the correlation errors in memory channels can lower the performance of the entanglement fidelity of standard QECCs (see [12], [18]). But if we consider the extra burst error correction abilities of them, they can indeed outperform the best QECCs of the same lengths for random 
errors.

\section{TheORY OF QuANTUM BURST ERROR CORRECTION CODES}

In this section, we introduce background of QECCs and develop the stabilizer formalism for QBECCs.

\section{A. Quantum Burst Error Correction Codes}

In a two-dimensional complex Hilbert space $\mathbb{C}^{2}$, a qubit $|v\rangle$ can be written as $|v\rangle=\alpha|0\rangle+\beta|1\rangle$, where $\alpha$ and $\beta$ are complex numbers satisfying $|\alpha|^{2}+|\beta|^{2}=1$. Two states $|v\rangle$ and $e^{i \theta}|v\rangle$, that are different up to a global phase $e^{i \theta}$, are considered to be the same in this paper. The Pauli matrices

$$
I_{2}=\left[\begin{array}{ll}
1 & 0 \\
0 & 1
\end{array}\right], X=\left[\begin{array}{ll}
0 & 1 \\
1 & 0
\end{array}\right], Z=\left[\begin{array}{cc}
1 & 0 \\
0 & -1
\end{array}\right], Y=\left[\begin{array}{cc}
0 & -i \\
i & 0
\end{array}\right]
$$

form a basis of the linear operators on $\mathbb{C}^{2}$, where $i=\sqrt{-1}$ and $Y=i X Z$. An $n$-qubit $|\psi\rangle$ is then a quantum state in the $n$-th tensor product of $\mathbb{C}^{2}$, i.e., $|\psi\rangle \in \mathbb{C}^{2^{n}} \equiv \mathbb{C}^{2} \otimes \mathbb{C}^{2} \otimes \cdots \otimes \mathbb{C}^{2}$.

Since it is possible to discretize quantum errors [19], [20], we only need to consider a discrete set of quantum errors of $n$ qubits, described by the following error group

$$
\mathcal{G}_{n}=\left\{i^{\lambda} w_{1} \otimes \cdots \otimes w_{n} \mid 0 \leq \lambda \leq 3, w_{i} \in I_{2}, X, Y, Z\right\} .
$$

Furthermore, it is sufficient to consider the quotient group $\overline{\mathcal{G}}_{n}=\mathcal{G}_{n} /\{ \pm 1, \pm i\}$ of $\mathcal{G}_{n}$ since the global phase $i^{\lambda}$ in $\mathcal{G}_{n}$ is not important. Let $\bar{e}=w_{1} \otimes w_{2} \otimes \cdots \otimes w_{n} \in \overline{\mathcal{G}}_{n}$ and $e=i^{\lambda} \bar{e} \in \mathcal{G}_{n}$. We define the burst length of $\bar{e}$ to be $\ell$, denoted by $b_{Q}(e)=\operatorname{bl}_{Q}(\bar{e})=\ell$, if the nonidentity matrices in $\bar{e}$ are confined to at most $\ell$ consecutive $w_{i}$ 's.

The idea of a QECC is to encode quantum information into a subspace of some larger Hilbert space. An $((n, K))$ QECC $Q$ is defined to contain the subspace of dimension $K$ in $\mathbb{C}^{2^{n}}$. If $K=2^{k}$, then $Q$ is also written as $Q=[[n, k]]$. A QECC $Q$ can correct arbitrary errors from an error class $\varepsilon$ [21], [22] if

$$
\left\langle c_{i}\left|E E^{\prime}\right| c_{j}\right\rangle=a_{\left(E, E^{\prime}\right)} \delta_{i j}
$$

for all $\left\langle c_{i} \mid c_{j}\right\rangle=\delta_{i j}$ and for all $E \neq E^{\prime} \in \varepsilon$, where $\left|c_{i}\right\rangle$ and $\left|c_{j}\right\rangle \in Q$, and $a_{\left(E, E^{\prime}\right)}$ is a constant which depends only on $E$ and $E^{\prime}$. If $\left\langle c_{i}\left|E E^{\prime}\right| c_{j}\right\rangle=0$ for all $\left|c_{i}\right\rangle,\left|c_{j}\right\rangle \in Q$ and for all $E \neq E^{\prime} \in \varepsilon$, then $Q$ is called a nondegenerate quantum code.

The above error-correcting condition (2) can be generalized to the burst error case.

Proposition 1: The code $Q$ can correct any quantum burst errors of length $l$ or less if and only if

$$
\left\langle c_{i}\left|E E^{\prime}\right| c_{j}\right\rangle=a_{\left(E, E^{\prime}\right)} \delta_{i j}
$$

for all $\left\langle c_{i} \mid c_{j}\right\rangle=\delta_{i j}$ and for all $\mathrm{bl}(E), \operatorname{bl}\left(E^{\prime}\right) \leq \ell$, where $\left|c_{i}\right\rangle$ and $\left|c_{j}\right\rangle \in Q, E$ and $E^{\prime} \in \mathcal{G}_{n}$, and $a_{\left(E, E^{\prime}\right)}$ is a constant which depends only on $E$ and $E^{\prime}$.

If $\left\langle c_{i}\left|E E^{\prime}\right| c_{j}\right\rangle=0$ for all $\left|c_{i}\right\rangle,\left|c_{j}\right\rangle \in Q$ and for all $\mathrm{bl}(E), \mathrm{bl}\left(E^{\prime}\right) \leq \ell$, where $E \neq E^{\prime} \in \mathcal{G}_{n}$, then $Q$ is a nondegenerate QBECC.

According to the group theoretic framework for QECCs in [4], [23], we can also get the stabilizer formalism for QBECCs.
Let $(a \mid b)$ and $\left(a^{\prime} \mid b^{\prime}\right)$ be two vectors in $\mathbb{F}_{2}^{2 n}$, the symplectic inner product of them is given by

$$
\left((a \mid b),\left(a^{\prime} \mid b^{\prime}\right)\right)_{s}=a \cdot b^{\prime}+a^{\prime} \cdot b .
$$

For a subspace $C$ of $\mathbb{F}_{2}^{2 n}$, the symplectic dual space $C^{\perp_{s}}$ of $C$ is given by

$$
C^{\perp_{s}}=\left\{u \in \mathbb{F}_{2}^{2 n} \mid \forall c \in C,(u, c)_{s}=0\right\} .
$$

We define the symplectic burst length of a nonzero vector $(a \mid b)=\left(a_{1} \cdots a_{n} \mid b_{1} \cdots b_{n}\right) \in \mathbb{F}_{2}^{2 n}$ to be the largest integer $1 \leq \ell \leq n$ such that $\left(a_{i} \mid b_{i}\right) \neq(0,0)$ and $\left(a_{i+\ell-1} \mid b_{i+\ell-1}\right) \neq$ $(0,0)$ for some $1 \leq i \leq n$. We denote by bl $\mathrm{b}_{s}((a \mid b))=\ell$.

According to [23], each element $E \in \mathcal{G}_{n}$ can be written uniquely as $E=i^{\lambda} X(a) Z(b)$ where $1 \leq \lambda \leq 3, X(a)|\psi\rangle=$ $|\psi+a\rangle, Z(b)|\psi\rangle=(-1)^{b \cdot \psi}|\psi\rangle$, and for $a, b \in \mathbb{F}_{2}^{n}$. It is easy to verify that $\mathrm{bl}_{Q}(E)=\mathrm{bl}_{Q}(\bar{E})=\mathrm{bl}_{s}((a \mid b))$. Then we have the group framework for quantum burst error correction codes.

Theorem 1: Suppose that there exists an $(n-k)$-dimensional linear subspace $C$ of $\mathbb{F}_{2}^{2 n}$ which is contained in its symplectic dual $C^{\perp_{s}}$, i.e., $C \subseteq C^{\perp_{s}}$. Let $\ell$ be the largest integer such that for arbitrary two vectors $e_{1} \neq e_{2} \in \mathbb{F}_{2}^{2 n}$ whose symplectic burst length $\leq \ell$ there is $e_{1}+e_{2} \notin C^{\perp_{s}} \backslash C$. Then there exists a quantum burst error correction code $Q=[[n, k]]$ which can correct arbitrary quantum burst errors of length $\ell$ or less. If all the $e_{1}+e_{2} \notin C^{\perp_{s}} \backslash\{0\}$, then $Q$ is a nondegenerate quantum burst error correction code.

Proof: The existence of the quantum code $Q$ has been shown in [23, Theorem 1]. The burst error correction abilities of $Q$ can be obtained directly by combining [23, Lemma 1] and Proposition 1.

As shown in [23], binary quantum codes can be constructed by using additive codes over $\mathbb{F}_{4}$. Define the trace inner product of two vectors $u, v \in \mathbb{F}_{4}^{n}$ by

$$
(u, v)_{t r}=\sum_{i=1}^{n}\left(u_{i} v_{j}^{2}+u_{i}^{2} v_{j}\right)
$$

Let $C$ be an additive code over $\mathbb{F}_{4}$, then the trace dual of $C$ with respect to the trace inner product is defined by

$$
C^{\perp_{t r}}=\left\{v \in \mathbb{F}_{4}^{n} \mid \forall u \in C,(u, v)_{t r}=0\right\} .
$$

Then Theorem 1 can also be reformulated by using additive codes over $\mathbb{F}_{4}$ and by replacing the symplectic inner product with trace inner product.

Theorem 2: Suppose that $C$ is an additive code over $\mathbb{F}_{4}$ which is contained in its trace dual $C^{\perp_{t r}}$, i.e., $C \subseteq C^{\perp_{t r}}$. Let $\ell$ be the largest integer such that for arbitrary two vectors $e_{1} \neq$ $e_{2} \in \mathbb{F}_{4}^{n}$ whose burst length $\leq \ell$ there is $e_{1}+e_{2} \notin C^{\perp_{t r}} \backslash C$. Then there exists a binary quantum burst error correction code $Q=[[n, k]]$ which can correct arbitrary quantum burst errors of length $\ell$ or less. If all the $e_{1}+e_{2} \notin C^{\perp_{t r}} \backslash\{0\}$, then $Q$ is a nondegenerate quantum burst error correction code.

The CSS code construction [2], [3] provides a direct way to construct QECCs from classical linear codes. The CSS construction for QBECCs can be obtained from Theorem 1.

Corollary 1 (CSS Construction): Let $C_{1}=\left[n, k_{1}\right]$ and $C_{2}=$ $\left[n, k_{2}\right]$ be two binary linear codes which have $\ell_{1}$ and $\ell_{2}$ burst error correction abilities, respectively, and such that $C_{2}^{\perp} \subseteq C_{1}$. 
Let $\ell$ be the largest integer such that for arbitrary two vectors $e_{1} \neq e_{2} \in \mathbb{F}_{2}^{n}$ whose burst length $\leq \ell$ there is $e_{1}+e_{2} \notin$ $\left(C_{1} \backslash C_{2}^{\perp}\right) \cup\left(C_{2} \backslash C_{1}^{\perp}\right)$. Then there exists a binary quantum burst error correction code $Q=\left[\left[n, k_{1}+k_{2}-n\right]\right]$ which can correct arbitrary quantum burst errors of length $\ell$ or less and if $\ell=\min \left\{\ell_{1}, \ell_{2}\right\}$, then $Q$ is a nondegenerate code.

\section{B. Quantum Reiger Bound}

For a classical code $C=[n, k]$ which can correct $\leq t$ random errors or can correct any burst errors of length $\leq \ell$, there exists two important upper bounds called the Singleton bound $n-k \geq 2 t$ and the Reiger bound $n-k \geq 2 \ell$ that constrain the random error correction and burst error correction abilities of $C$, respectively (see [1]). In quantum codes, let $Q=[[n, k]]$ be a QECC which can correct $\leq t$ quantum random errors, there exists the quantum Singleton bound $n-k \geq 4 t$ which is an upper bound for the quantum random error correction ability of code $Q$ (see [19], [23]).

In the following, we derive the quantum Reiger bound (QRB) which is an upper bound for the quantum burst error correction ability of code $Q$.

Theorem 3 (Quantum Reiger Bound): If an [[n, $k]]$ QBECC $Q$ can correct quantum burst errors of length $\ell$, then it satisfies

$$
n-k \geq 4 \ell .
$$

Proof: The proof follows closely by that of the quantum Singleton bound given by Preskill (see [24, p.32] and [19, p.568]).

First of all, Lemma 1 in the Appendix says that if $Q$ can correct $\ell$ burst errors, then it must satisfy $n>4 \ell$, a consequence following from the quantum no-cloning principle.

Then we introduce a $k$-qubit ancilla system $A$, and construct a pure state $|\Psi\rangle_{A Q}$ that is maximally entangled between the system $A$ and the $2^{k}$ codewords of the $[[n, k]]$ QBECC $Q$ :

$$
|\Psi\rangle_{A Q}=\frac{1}{\sqrt{2^{k}}} \sum|x\rangle_{A}|x\rangle_{Q}
$$

where $\left\{|x\rangle_{A}\right\}$ denotes an orthonormal basis for the $2^{k}$ dimensional Hilbert space of the ancilla, and $\left\{|x\rangle_{Q}\right\}$ denotes an orthonormal basis for the $2^{k}$-dimensional code subspace. It is obvious that

$$
S(A)_{\Psi}=k=S(Q)_{\Psi},
$$

where $S(A)_{\rho}=-\operatorname{Tr} \rho_{A} \log \rho_{A}$ is the von Neumann entropy of a density operator $\rho_{A}$.

Next we divide the $n$-qubit QBECC $Q$ into three disjoint parts so that $Q^{(1)}$ and $Q^{(2)}$ consist of $2 \ell$ qubits each and $Q^{(3)}$ consists of the remaining $n-4 \ell$ qubits. If we trace out $Q^{(2)}$ and $Q^{(3)}$, the reduced density matrix that we obtained must contain no correlations between $Q^{(1)}$ and the ancilla $A$, a consequence following from Lemma 2 in the Appendix. This means that the entropy of system $A Q^{(1)}$ is additive:

$$
S\left(Q^{(2)} Q^{(3)}\right)_{\Psi}=S\left(A Q^{(1)}\right)_{\Psi}=S(A)_{\Psi}+S\left(Q^{(1)}\right)_{\Psi} .
$$

Similarly,

$$
S\left(Q^{(1)} Q^{(3)}\right)_{\Psi}=S\left(A Q^{(2)}\right)_{\Psi}=S(A)_{\Psi}+S\left(Q^{(2)}\right)_{\Psi} .
$$

Furthermore, in general, the von Neumann entropy is subadditive, so that

$$
\begin{gathered}
S\left(Q^{(1)} Q^{(3)}\right)_{\Psi} \leq S\left(Q^{(1)}\right)_{\Psi}+S\left(Q^{(3)}\right)_{\Psi} \\
S\left(Q^{(2)} Q^{(3)}\right)_{\Psi} \leq S\left(Q^{(2)}\right)_{\Psi}+S\left(Q^{(3)}\right)_{\Psi}
\end{gathered}
$$

Combining these inequalities with the equalities above, we find

$$
\begin{gathered}
S(A)+S\left(Q^{(2)}\right)_{\Psi} \leq S\left(Q^{(1)}\right)_{\Psi}+S\left(Q^{(3)}\right)_{\Psi} \\
S(A)+S\left(Q^{(1)}\right)_{\Psi} \leq S\left(Q^{(2)}\right)_{\Psi}+S\left(Q^{(3)}\right)_{\Psi}
\end{gathered}
$$

Both inequalities can be simultaneously satisfied only if

$$
S(A)_{\Psi} \leq S\left(Q^{(3)}\right)_{\Psi}
$$

Finally, we have

$$
S(A)_{\Psi}=k \leq n-4 \ell,
$$

since $S\left(Q^{(3)}\right)$ is bounded above by its dimension $n-4 \ell$. We then conclude the quantum Reiger bound.

\section{CONSTRUCTION OF QUANTUM BURST ERror CORRECTION CODES}

In this section we provide two methods for constructing QBECCs: one by using computer search based on the stabilizer formalism, and the other by concatenating and interleaving quantum tensor product codes.

\section{A. Stabilizer QBECCs Constructed by Using Computer Search}

We create a program using Magma software (version V2.1216) to search all possible cyclic codes to a reasonable length ( $n \leq 41$ ) according to Proposition 1 and make the codes as close as possible to the quantum Reiger bound. For simplicity, we only consider the construction of QBECCs from cyclic codes with odd length. We list new codes that are near or saturate the quantum Reiger bound found by this program in Table I. The bold numbers " $1-\mathbf{3}$ " stand for the coefficients and the superscript numbers stand for the exponents in the generator polynomials of the corresponding classical cyclic codes [1]. Notice that the burst error-correcting abilities of most QBECCs in Table I can saturate the quantum Reiger bound. In particular, we get several degenerate QBECCs which are the first class of QBECCs until now and they can saturate the quantum Reiger bound. Moreover, some of the constructed QBECCs are better than the QBECCs in Ref. [10], e.g., the codes [[15,3]], [[35, 17]] and [[35, 13]] in Table I have larger dimensions than the codes $[[15,2]]$, [[35, 14]] and $[[35,11]]$ in Ref. [10], respectively, but have the same burst error-correcting ability.

We remark that some QBECCs of length up to 51 have been found by using computer search in Ref. [10]. However, only CSS type QBECCs were considered and no degenerate codes were obtained in Ref. [10]. 
TABLE I

COMPUTER SEARCHING FOR QUANTUM BURST ERROR CORRECTION CODES

\begin{tabular}{|c|c|c|c|c|}
\hline$[n, k]]$ & $l$ & Generator Polynomials & QRB & Degenerate? \\
\hline$[13,1]]$ & 3 & $g=\left(\mathbf{1}^{6} \mathbf{2}^{5} \mathbf{3}^{3} \mathbf{2}^{1} \mathbf{1}^{0}\right)$ & 3 & False \\
\hline$[15,3]]$ & 3 & $g=\left(\mathbf{1}^{6} \mathbf{2}^{3} \mathbf{1}^{0}\right)$ & 3 & False \\
\hline$[17,1]]$ & 4 & $g=\left(\mathbf{1}^{8} \mathbf{3}^{7} \mathbf{1}^{6} \mathbf{1}^{5} \mathbf{2}^{4} \mathbf{1}^{3} \mathbf{1}^{2} \mathbf{3}^{1} \mathbf{1}^{0}\right)$ & 4 & False \\
\hline$[17,1]]$ & 4 & $g=\left(\mathbf{1}^{8} \mathbf{3}^{7} \mathbf{3}^{5} \mathbf{3}^{4} \mathbf{3}^{3} \mathbf{3}^{1} \mathbf{1}^{0}\right)$ & 4 & True \\
\hline$[[21,9]]$ & 2 & $\begin{array}{l}g_{1}=\left(\mathbf{1}^{6} \mathbf{1}^{4} \mathbf{1}^{1} \mathbf{1}^{0}\right) \\
g_{2}=\left(\mathbf{1}^{6} \mathbf{1}^{4} \mathbf{1}^{2} \mathbf{1}^{1} \mathbf{1}^{0}\right)\end{array}$ & 3 & False \\
\hline$[[23,1]]$ & 5 & $\left(g=\mathbf{1}^{11} \mathbf{1}^{9} \mathbf{1}^{7} \mathbf{1}^{6} \mathbf{1}^{5} \mathbf{1}^{1} \mathbf{1}^{0}\right)$ & 5 & False \\
\hline$[25,1]]$ & 6 & $g=\left(\mathbf{1}^{12} \mathbf{2}^{11} \mathbf{1}^{10} \mathbf{2}^{7} \mathbf{3}^{6} \mathbf{2}^{5} \mathbf{1}^{2} \mathbf{2}^{1} \mathbf{1}^{0}\right)$ & 6 & True \\
\hline$[[25,5]]$ & 5 & $g=\left(\mathbf{1}^{10} \mathbf{2}^{5} \mathbf{1}^{0}\right)$ & 5 & False \\
\hline$[[29,1]]$ & 7 & $\begin{array}{r}g=\left(\mathbf{1}^{14} \mathbf{2}^{13} \mathbf{2}^{11} \mathbf{3}^{10} \mathbf{1}^{9} \mathbf{3}^{8}\right. \\
\left.\mathbf{2}^{7} \mathbf{3}^{6} \mathbf{1}^{5} \mathbf{3}^{4} \mathbf{2}^{3} \mathbf{2}^{1} \mathbf{1}^{0}\right)\end{array}$ & 7 & True \\
\hline$[[35,25]]$ & 2 & $g=\left(\mathbf{1}^{5} \mathbf{2}^{4} \mathbf{3}^{2} \mathbf{2}^{1} \mathbf{1}^{0}\right)$ & 2 & False \\
\hline$[[35,19]]$ & 3 & $g=\left(\mathbf{1}^{8} \mathbf{2}^{7} \mathbf{3}^{6} \mathbf{1}^{5} \mathbf{3}^{3} \mathbf{1}^{2} \mathbf{1}^{1} \mathbf{1}^{0}\right)$ & 4 & False \\
\hline$[[35,17]]$ & 4 & $g=\left(\mathbf{1}^{9} \mathbf{3}^{7} \mathbf{3}^{6} \mathbf{3}^{5} \mathbf{3}^{4} \mathbf{2}^{3} \mathbf{2}^{2} \mathbf{2}^{1} \mathbf{1}^{0}\right)$ & 4 & False \\
\hline$[[35,13]]$ & 5 & $g=\left(\mathbf{1}^{11} \mathbf{3}^{10} \mathbf{2}^{9} \mathbf{1}^{8} \mathbf{2}^{7} \mathbf{2}^{6} \mathbf{3}^{5} \mathbf{1}^{3} \mathbf{2}^{2} \mathbf{1}^{1} \mathbf{1}^{0}\right)$ & 5 & False \\
\hline$[[35,7]]$ & 6 & $g=\left(\begin{array}{rl}\mathbf{1}^{14} \mathbf{2}^{13} \mathbf{3}^{11} \mathbf{2}^{10} \mathbf{2}^{9} \mathbf{3}^{8} \\
\left.\mathbf{1}^{7} \mathbf{1}^{6} \mathbf{2}^{5} \mathbf{3}^{3} \mathbf{2}^{2} \mathbf{3}^{1} \mathbf{1}^{0}\right)\end{array}\right.$ & 7 & False \\
\hline$[[41,1]]$ & 10 & 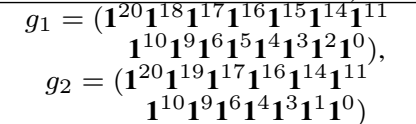 & 10 & True \\
\hline
\end{tabular}

\section{B. Concatenation Construction of QBECCs Based on Quan- tum Tensor Product Codes}

In this section we give a concatenation construction of long QBECCs from two short component codes based on the quantum tensor product codes structure [7], [25].

Firstly we present a brief review of classical and quantum tensor product codes. Details could be found in, e.g., [7], [26].

Let $C_{1}=\left[n_{1}, k_{1}\right]_{2}$ be a linear code with a parity check matrix $H_{1}$ and let $\rho_{1}=n_{1}-k_{1}$ be the number of check symbols. Let $C_{2}=\left[n_{2}, k_{1}\right]_{2^{\rho_{1}}}$ be a linear code over the extension filed $\mathbb{F}_{2^{\rho_{1}}}$ with a parity check matrix $H_{2}$. Then the tensor product code (TPC) of $C_{1}$ and $C_{2}$ is denoted by $\mathcal{C}=C_{2} \otimes_{H} C_{1}$, and the parity check matrix $\mathcal{C}$ is given by

$$
H=H_{2} \otimes H_{1} .
$$

By selecting different types of component codes, TPCs can be designed to provide different error control abilities.

In [7], a framework for the construction of quantum tensor product codes (QTPC), which can provide a wide variety of quantum error-correcting, error-detecting or error-locating properties, was proposed. In particular, if one of the component codes is selected as a BECC, then QTPCs can have multiple quantum burst error-correcting abilities, but provided these bursts fall in distinct subblocks.

Theorem 4 ([7]): Let $C_{1}=\left[n_{1}, k_{1}\right]_{q}$ be an $\ell_{1}$ burst error correction code, and let $C_{2}=\left[n_{2}, k_{2}\right]_{q^{\rho_{1}}}$ be an $\ell_{2}$ burst error correction code over the extension field $\mathbb{F}_{q^{\rho_{1}}}$, and the numbers of check symbols are $\rho_{1}=n_{1}-k_{1}$ and $\rho_{2}=n_{2}-k_{2}$, respectively. If $q=2$ and $C_{1}^{\perp} \subseteq C_{1}$ or if $q=4$ and $C_{1}^{\perp_{h}} \subseteq C_{1}$, where $C_{1}^{\perp_{h}}$ is the Hermitian dual code of $C_{1}$, then there exists a QTPC $\mathcal{Q}=\left[\left[n_{1} n_{2}, n_{1} n_{2}-2 \rho_{1} \rho_{2}\right]\right]$ which can correct $\ell_{2}$ or fewer bursts of burst errors each is a burst of length $\ell_{1}$ or less, provided these bursts fall in distinct subblocks.

Although we can use QTPCs to correct a single burst of errors since QTPCs have multiple burst error correction

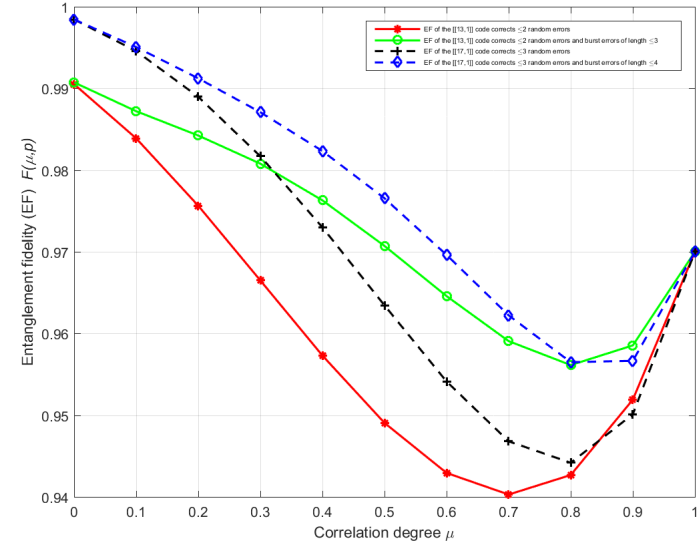

Fig. 1. The entanglement fidelity (EF) of the two $[[13,1]]$ and $[[17,1]]$ codes with respect to the correlation degree $0 \leq \mu \leq 1$, the error probability is set to be $p=3 \times 10^{-2}$.

abilities, they are not efficient enough any more. To overcome this problem, we can interleave the encoded qubits before sending into the quantum channels, and deinterleave after receiving the qubits. The whole interleaving/deinterleaving procedure is summarized as follows:

(1). After the quantum encoding, we arrange the encoded $n_{1} n_{2}$ qubits into an $n_{1} \times n_{2}$ code array.

(2). Instead of transmitting the encoded qubits sequentially one by one, we do an interleaved transmission. Denote by $\ell_{1}$ the burst error correction ability of $C_{1}$. If $\ell_{1} \mid n_{1}$ and the component code $C_{2}$ can correct end-around (see [1]) burst errors, then we divide the $n_{1} \times n_{2}$ code array into $s=n_{1} / \ell_{1}$ subblocks by rows. We do the transmission subblock by subblock, and in each subblock, we transmit the qubits column by column sequentially (each column contains $\ell_{1}$ qubits).

(3). After receiving all the $n_{1} n_{2}$ qubits, we deinterleave the qubits into an $n_{1} \times n_{2}$ code array so that the quantum decoding can be processed next, and the deinterleaving is just the inverse of the interleaving. The deinterleaving/interleaving procedure can be accomplished by using quantum SWAP gates (see [19]).

Suppose that a single burst errors of length at most $\ell_{1} \ell_{2}$ happens among the $n_{1} n_{2}$ interleaved qubits. After the quantum transmition and deinterleaving, the $n_{1} n_{2}$ qubits are recovered to their original positions, but the single burst errors of length at most $\ell_{1} \ell_{2}$ has been dispersed into $\ell_{2}$ or fewer consecutive subblocks (end around) and each subblock contains a burst errors of length at most $\ell_{1}$. Thus the resultant QTPC $\mathcal{Q}$ can correct a single burst error of length at most $\ell_{1} \ell_{2}$ according to Theorem 4 and Ref. [7]. Then we have the following result.

Theorem 5: Let $C_{1}=\left[n_{1}, k_{1}\right]_{q}$ and $C_{2}=\left[n_{2}, k_{2}\right]_{q^{\rho_{1}}}$ be two component codes of a QTPC with parameters $\mathcal{Q}=$ [ $\left.\left[n_{1} n_{2}, n_{1} n_{2}-2 \rho_{1} \rho_{2}\right]\right]$, where $C_{1}$ is an $\ell_{1}$ burst error correction code and $C_{2}$ is an $\ell_{2}$ burst error correction code (end-around), and the numbers of check symbols are $\rho_{1}=n_{1}-k_{1}$ and $\rho_{2}=$ $n_{2}-k_{2}$, respectively. If $\ell_{1} \mid n_{1}$, then there exists an $\ell_{1} \ell_{2}$ burst error correction quantum code $\mathcal{Q}=\left[\left[n_{1} n_{2}, n_{1} n_{2}-2 \rho_{1} \rho_{2}\right]\right]$. 


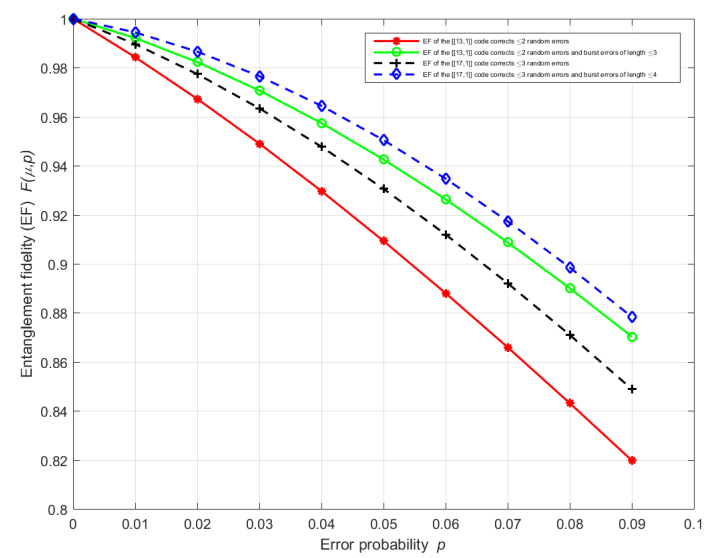

Fig. 2. The entanglement fidelity (EF) of the two $[[13,1]]$ and $[[17,1]]$ codes with respect to the error probability $1 \times 10^{-5} \leq p \leq 1 \times 10^{-1}$, the correlation degree is set to be $\mu=0.5$.

Example 1: We choose $C_{1}=[15,9]_{4}$ as a 3 burst error correction cyclic code with the generator polynomial $g=\left(\mathbf{1}^{6} \mathbf{2}^{3} \mathbf{1}^{0}\right)$ and it is Hermitian dual containing by Table I. Let $C_{2}=\left[n_{2}, n_{2}-2 \ell_{2}, 2 \ell_{2}+1\right]_{4^{6}}$ be an MDS code over the extension field $\mathbb{F}_{4^{6}}$ with $2 \leq n_{2} \leq 4^{6}+2$ and $1 \leq \ell_{2} \leq\left\lfloor\frac{n_{2}-1}{2}\right\rfloor$. Then there exists a $3 \ell_{2}$ burst error correction QTPC with parameters $\mathcal{Q}=\left[\left[15 n_{2}, 15 n_{2}-24 \ell_{2}\right]\right]$.

\section{Performance of QbeCCs over Markovian CORRELATED QuANTUM MEMORY CHANNELS}

In this section we evaluate the performance of two specific QBECCs in the presence of correlated errors.

The channel model that we choose is a Markovian correlated depolarizing quantum channel [12], [18]:

$$
\begin{aligned}
\Phi^{(n)}(\rho)= & \sum_{i_{1}, \ldots, i_{n}=0}^{3} p_{i_{n} \mid i_{n-1}}^{(n)} \cdots p_{i_{2} \mid i_{1}}^{(2)} p_{i_{1}}^{(1)} \\
& \times E_{i_{n}}^{(n)} \cdots E_{i_{1}}^{(1)} \rho E_{i_{1}}^{(1) \dagger} \cdots E_{i_{n}}^{(n) \dagger},
\end{aligned}
$$

where $\left\{E_{i_{j}}^{(j)}\right\}_{i, j}$ are the Pauli operators, and the conditional probabilities satisfy the normalization condition

$$
\sum_{i_{1}, \ldots, i_{n}=0}^{3} p_{i_{n} \mid i_{n-1}}^{(n)} \cdots p_{i_{2} \mid i_{1}}^{(2)} p_{i_{1}}^{(1)}=1,
$$

where $p_{l \mid k}^{(j)}=(1-\mu) p_{l}+\mu \delta_{(k, l)}$ for $1 \leq j \leq n$ and $0 \leq k, l \leq$ 3 , and $p_{0}=1-p, p_{1,2,3}=p / 3$ are the error probabilities in the depolarizing channel, $\mu \in[0,1]$ is the correlation degree.

Specifically, we show the performance of the two specific codes by measuring the entanglement fidelity $\mathcal{F}(p, \mu)$ as a function of the error probability $p$ and the correlation degree $\mu$ [19].

The two specific codes considered here are two QBECCs $[[13,1]]$ and $[[17,1]]$ in Table I which can correct burst errors of length $\leq 3$ and of length $\leq 4$, respectively. Through the computation, we know that the minimum distances of the two codes are 5 and 7 , then they can also correct $\leq 2$ and $\leq 3$ random errors, respectively, and they have achieved the upper

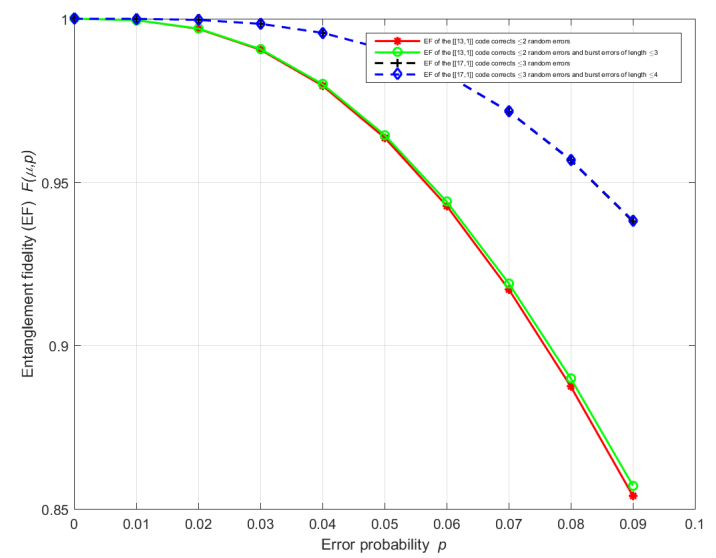

Fig. 3. The entanglement fidelity (EF) of the two $[[13,1]]$ and $[[17,1]]$ codes with respect to the error probability $1 \times 10^{-5} \leq p \leq 1 \times 10^{-1}$, the correlation degree is set to be $\mu=0$.

bounds in Ref. [27]. We plot the performance by means of entanglement fidelity of the two specific codes with respect to random errors or burst errors, versus the correlation degree $\mu$ or the error probability $p$ in Fig. 1 or Fig. 2, respectively. For details about the computation of the entanglement fidelity, see [12], [18], [19], and the computation results are put in the Cloud.

It is shown that the correlation errors do degrade the performance of the entanglement fidelity of the two codes in Fig. 1. If the the correlation degree $\mu=0$ which means that errors are independent with each other, then the extra burst error correction abilities of the two codes do little help to improve the performance of entanglement fidelity of them, respectively, see Fig. 1 and Fig. 3. However, if we consider the correlated errors when $0<\mu<1$, the performance of the entanglement fidelity can be improved largely, see Fig. 1 and Fig. 2. In particular, in Fig. 2, the [[13, 1]] code have better performance when considering its extra burst error correction ability compared to the $[[17,1]]$ code when only considering the random error correction ability.

\section{ACKNOWLEDGMENT}

J. Fan was supported by NJIT (Grant No. YKJ201719) and by NSFC (Grant No. 61403188). M.-H. Hsieh was supported in part by an ARC Future Fellowship under Grant FT140100574 and in part by U.S. the Army Research Office for Basic Scientific Research under Grant W911NF-17-10401.

\section{REFERENCES}

[1] S. Lin and D. J. Costello, Error Control Coding: Fundamentals and Applications, 2nd ed. Upper Saddle River, NJ: Prentice-Hall, Inc., 2004.

[2] A. M. Steane, "Error correcting codes in quantum theory," Phys. Rev. Lett., vol. 77, no. 5, p. 793, 1996.

[3] A. R. Calderbank and P. W. Shor, "Good quantum error-correcting codes exist," Phys. Rev. A, vol. 54, no. 2, p. 1098, 1996.

[4] A. R. Calderbank, E. M. Rains, P. W. Shor, and N. J. Sloane, "Quantum error correction and orthogonal geometry," Phys. Rev. Lett., vol. 78, no. 3, p. 405, 1997. 
[5] T. Brun, I. Devetak, and M.-H. Hsieh, "Correcting quantum errors with entanglement," Science, vol. 314, no. 5798, pp. 436-439, 2006.

[6] M. M. Wilde, M. H. Hsieh, and Z. Babar, "Entanglement-assisted quantum turbo codes," IEEE Trans. Inf. Theory, vol. 60, no. 2, pp. $1203-$ 1222, Feb 2014

[7] J. Fan, Y. Li, M.-H. Hsieh, and H. Chen, "On quantum tensor product codes," Quantum Inf. Comput., vol. 17, no. 13\&14, pp. 1105-1122, 2017.

[8] F. Vatan, V. P. Roychowdhury, and M. Anantram, "Spatially correlated qubit errors and burst-correcting quantum codes," IEEE Trans. Inf. Theory, vol. 45, no. 5, pp. 1703-1708, 1999.

[9] S. Kawabata, "Quantum interleaver: quantum error correction for burst error," J. Phys. Soc. Jpn., vol. 69, no. 11, pp. 3540-3543, 2000.

[10] K.-i. Tokiwa, K. Kiyama, T. Yamasaki et al., "Some binary quantum codes with good burst-error-correcting capabilities," 2005.

[11] D. Kretschmann and R. F. Werner, "Quantum channels with memory," Phys. Rev. A, vol. 72, p. 062323, Dec 2005.

[12] F. Caruso, V. Giovannetti, C. Lupo, and S. Mancini, "Quantum channels and memory effects," Rev. Mod. Phys., vol. 86, no. 4, p. 1203, 2014.

[13] C. Y. Lai, M. H. Hsieh, and H. F. Lu, "On the macwilliams identity for classical and quantum convolutional codes," IEEE Trans. Commun., vol. 64 , no. 8, pp. 3148-3159, Aug 2016.

[14] Y. Fujiwara and V. D. Tonchev, "A characterization of entanglementassisted quantum low-density parity-check codes," IEEE Trans. Inf. Theory, vol. 59, no. 6, pp. 3347-3353, June 2013.

[15] M. H. Hsieh, W. T. Yen, and L. Y. Hsu, "High performance entanglement-assisted quantum ldpc codes need little entanglement," IEEE Trans. Inf. Theory, vol. 57, no. 3, pp. 1761-1769, March 2011.

[16] M.-H. Hsieh, T. A. Brun, and I. Devetak, "Entanglement-assisted quantum quasicyclic low-density parity-check codes," Phys. Rev. A, vol. 79, p. 032340, Mar 2009

[17] M.-H. Hsieh, I. Devetak, and T. Brun, "General entanglement-assisted quantum error-correcting codes," Phys. Rev. A, vol. 76, p. 062313, Dec 2007.

[18] C. Cafaro and S. Mancini, "Quantum stabilizer codes for correlated and asymmetric depolarizing errors," Phys. Rev. A, vol. 82, no. 1, p. 012306 , 2010.

[19] M. A. Nielsen and I. L. Chuang, Quantum Computation and Quantum Information. Cambridge University Press, 2000, no. 2.

[20] T. A. Brun, I. Devetak, and M. H. Hsieh, "Catalytic quantum error correction," IEEE Trans. Inf. Theory, vol. 60, no. 6, pp. 3073-3089, June 2014.

[21] C. H. Bennett, D. P. DiVincenzo, J. A. Smolin, and W. K. Wootters, "Mixed-state entanglement and quantum error correction," Phys. Rev. A vol. 54, no. 5, p. 3824, 1996.

[22] E. Knill and R. Laflamme, "Theory of quantum error-correcting codes," Phys. Rev. A, vol. 55, no. 2, p. 900, 1997.

[23] A. Calderbank, E. Rains, P. Shor, and N. Sloane, "Quantum error correction via codes over GF(4)," IEEE Trans. Inf. Theory, vol. 44 no. 4, pp. 1369-1387, 1998.

[24] J. Preskill, "Physics 229: Advanced mathematical methods of physicsquantum computation and information," California Institute of Technology, 1998.

[25] J. Fan and H. Chen, "Comments on and corrections to "on the equivalence of generalized concatenated codes and generalized error location codes"," IEEE Trans. Inf. Theory, vol. 63, no. 8, pp. 5437-5439, 2017.

[26] J. K. Wolf, "On codes derivable from the tensor product of check matrices," IEEE Trans. Inf. Theory, vol. 11, no. 2, pp. 281-284, 1965.

[27] M. Grassl, "Bounds on the minimum distance of linear codes and quantum codes," Online available at http://www.codetables.de, 2007.

\section{APPENDIX}

Lemma 1 (No-Cloning Bound): For an arbitrary $\ell$ burst error correction code $C=[[n, k \geq 1]]$ exists only if

$$
n>4 \ell \text {. }
$$

Proof: Suppose that there exists a code $C^{\prime}=[[n, k \geq 1]]$ with $2 \leq n \leq 4 \ell$. After encoding $k$ qubits into $n$ ones, we split the encoded block into two sub-blocks, one contains the first $\left\lfloor\frac{n}{2}\right\rfloor$ qubits and the other contains the rest of the $n-\left\lfloor\frac{n}{2}\right\rfloor$ qubits.
If we append $\left\lfloor\frac{n}{2}\right\rfloor$ ancilla qubits $|0 \cdots 0\rangle$ to the first subblock, and append $n-\left\lfloor\frac{n}{2}\right\rfloor$ ancilla qubits $|0 \cdots 0\rangle$ to the second sub-block, then the original encoded block has spawned two offspring, the first one with located burst errors of length at most $\left\lfloor\frac{n}{2}\right\rfloor$, and the second one with located burst errors of length at most $n-\left\lfloor\frac{n}{2}\right\rfloor$. If we were able to correct the two located burst errors in each of the offspring (see Lemma 2), we would obtain two identical copies of the parent encoded block, which is a contradiction with the quantum no-cloning theorem [19]. Therefor we must have $n>4 \ell$.

Lemma 2 (Located Burst Errors): For a QECC $Q=[[n, k]]$ that corrects arbitrary burst errors of length $\ell$ or less can correct located burst errors of length at most $2 \ell$.

Proof: Denote an arbitrary error of length $n$ by

$$
e=e_{1} \otimes \ldots \otimes e_{x} \otimes \ldots \otimes e_{y} \otimes \ldots \otimes e_{n}
$$

where $1 \leq x<y \leq n, y-x+1=2 \ell$, and $e_{i}(1 \leq i \leq n)$ are Pauli matrices. The set $E(x, y)$ of burst errors to be corrected is the set of all Pauli operators, where each acts trivially on the qubits 1 to $x-1$ and on the qubits $y+1$ to $n$ (except $x=1$ and $y=n)$. Then each error in $E(x, y)$ has a burst length of at most $2 \ell$. But now, for each $E_{a}$ and $E_{b}$ in $E(x, y)$, the product $E_{a}^{\dagger} E_{b}$ also has a burst of length at most $2 \ell$. Therefore, the burst error-correcting criterion (3) is satisfied for all $E_{a, b} \in E$, provided $Q$ is an $\ell$ burst error correction code. 